features $(p=0.002$ for both). There were no other significant associations between S100 levels and other conventional markers such as C3, C4 and the anti-dsDNA antibodies.

Conclusions We demonstrate increased S100 proteins expression in SLE patients. Although they were not strongly associated with disease activity, S100 proteins and namely S100A4 could be proposed as a diagnostic biomarker for SLE.

Acknowledgements MHCR for conceptual development of research organisation 00023728 and SVV 260373.

Disclosure of interest None declared

\section{P059 EX VIVO COMPARISON OF BARICITINIB, UPADACITINIB, FILGOTINIB AND TOFACITINIB FOR CYTOKINE SIGNALLING IN HUMAN LEUKOCYTE SUBPOPULATIONS}

${ }^{1}$ IB Mclnnes, ${ }^{2} \mathrm{R}$ Higgs, ${ }^{2} \mathrm{~J}$ Lee, ${ }^{2} \mathrm{WL}$ Macias, ${ }^{2} \mathrm{~S} \mathrm{Na},{ }^{2} \mathrm{RA}$ Ortmann, ${ }^{2} \mathrm{G}$ Rocha, ${ }^{3} \mathrm{~T}$ Wehrman, ${ }^{2} \mathrm{X}$ Zhang, ${ }^{2} \mathrm{SH}$ Zuckerman, ${ }^{4} \mathrm{PC}$ Taylor, ${ }^{5} \mathrm{C}$ Perrier* ${ }^{1}$ 'Division of Immunology, Infection and Inflammation, University of Glasgow, Glasgow, UK; ${ }^{2}$ Eli Lilly and Company, Indianapolis; ${ }^{3}$ Primity Bio, Fremont, USA; ${ }^{4}$ Medical Sciences Division, University of Oxford, Oxford, UK; ${ }^{5}$ Presenting on behalf of Eli Lilly and Company, Indianapolis, USA

\subsection{6/annrheumdis-2018-EWRR2018.78}

Introduction Baricitinib (BARI), an oral selective Janus kinase (JAK) $1 / 2$ inhibitor, approved in the $\mathrm{EU}$ for moderate to severe active RA.

Objectives To compare in vitro cellular pharmacology of BARI to upadacitinib (ABT), filgotinib (FILGO), and tofacitinib (TOFA), three JAK inhibitors (JAKis) currently approved or in clinical development.

Methods Peripheral blood mononuclear cells from healthy donors $(n=6-12)$ were incubated with different JAKis. After cytokine stimulation, phosphorylated signal transducer and activator of transcription (pSTAT) levels were measured and $\mathrm{IC}_{50}$ calculated in phenotypically gated leukocyte subpopulations. Therapeutic dose relevance of in vitro analysis was assessed using calculated mean concentration-time (CT) profiles over 24 hour JAKi-treated subjects (BARI $4 \mathrm{mg}$ QD; ABT 15 and $30 \mathrm{mg}$ QD; FILGO 100 and $200 \mathrm{mg}$ QD; TOFA 5 and $10 \mathrm{mg}$ BID). Time above $\mathrm{IC}_{50}(\mathrm{~T}>\mathrm{IC} ; \mathrm{h} /$ day) and average daily $\%$ inhibition of pSTAT formation (\%SI) were calculated for each JAKi, cytokine, and cell type.

Results Tested cytokines did not signal in all cell types. When signalling was detected, $\mathrm{IC}_{50}, \% \mathrm{SI}$, and $\mathrm{T}>\mathrm{IC}$ for a particular JAKi exhibited similar dose dependent inhibition across cell types. For JAK1/3 dependent signalling across 4 cytokines (IL$2,4,15,21), \mathrm{IC}_{50}$ for $\mathrm{ABT}$ and TOFA were more potent than BARI; FILGO was the least potent. Overlaid on CT profiles, this indicated generally higher\%SI and longer $\mathrm{T}>\mathrm{IC}$ for ABT and TOFA compared to BARI and FILGO. For IL-6 (JAK1/2),\%SI and T>IC was TOFA $>$ BARI/ABT $>$ FILGO and for IL-10 (JAK1/TYK2),\%SI was TOFA $>$ BARI/ABT $>$ FILGO. IFN- $\gamma(\mathrm{JAK} 1 / 2)$ was modulated only by BARI, ABT, and TOFA. IFN- $\alpha$ (JAK1/TYK2) signalling was most potently inhibited by BARI and ABT. FILGO did not appear to modulate GM-CSF signalling (JAK2/2), while $\%$ SI and $\mathrm{T}>\mathrm{IC}$ were similar between BARI and ABT.

Conclusions JAKis modulate distinct cytokine pathways to differing degrees and durations over 24 hour. BARI and FILGO inhibited JAK1/3 signalling less than ABT and TOFA. No JAKis agent potently or continuously inhibited an individual cytokine signalling pathway throughout dosing interval, implying the varying efficacy and safety profiles of JAKis across disease states.

Acknowledgements
Study support Eli Lilly and Company and Incyte Corporation. Encore of ACR/ARHP-2017 Annual Scientific Meeting, Nov 4-8, 2016; San Diego, CA, USA.

Disclosure of interest None declared

\section{P060 IL-38 IN ARTHRITIS MATURATION AND DEGRADATION OF THIS NEW IL-1 FAMILY ANTI-INFLAMMATORY CYTOKINE}

M Harel, T Garraud, B Le Goff, F Blanchard*. 'INSERM UMR1238 Rheumatology unit, Nantes University Hospital, Nantes, France

\subsection{6/annrheumdis-2018-EWRR2018.79}

Introduction Interleukin 38 (IL-38) is an IL-1 family cytokine with potential anti-inflammatory activity. Recently we showed that over-expression of full length IL-38 reduces inflammation in arthritic mice by $20 \%-30 \%$, reduces macrophage infiltration and the expression of cytokines implicated in the biology of Th17 cells. ${ }^{1}$ However, IL-38 does not have a classical signal peptide, and it is unclear how it is released for example from monocytes/macrophages. It needs post-translational processing (N-terminal truncation) to acquire full biological activity, but the required proteases are unknown.

Objectives The aim is to study the maturation of IL-38 in the inflammatory process.

Methods THP1 macrophagic cells were transduced with a lentivirus encoding full length IL-38 to over-express this cytokine. Secretion and maturation (truncation) of endogenous or overexpressed IL-38 has been studied by western blot. The potential proteases implicated in IL-38 N-terminal truncation have been analysed in silico (Prosper webserver) and by biochemical tests using recombinant proteases and their inhibitors.

Results In the synovial fluid of RA patients, we observed 2 truncated forms of IL-38. To determine if apoptosis is a mechanism responsible for the maturation of IL-38, we tested different stress conditions on THP1 macrophages that overexpress IL-38. In conditioned media, three truncated forms of IL-38 were revealed by Western Blot under conditions of apoptosis, exacerbated oxidative stress, or necrosis. However, none of these conditioned media appears to have a high antiinflammatory effect on macrophages. Our results rather suggest that cell death by apoptosis or necrosis results in IL-38 degradation and loss of biological effect. In silico, several potential cleavage sites were found for calpain, MMP-2, MMP-9 and cathepsin G. Incubation of recombinant full length IL-38 with MMP-2 or cathepsin G leads to significant degradation of this cytokine. On apoptotic THP1 cells overexpressing IL-38, an inhibitor of MMPs and TACE (TAPI) also reduces the release and degradation of IL-38.

Conclusions The degradation and loss of the anti-inflammatory effect of IL-38 appears to occur during cell death in the extracellular space, and could involve MMPs. The processes involved in the maturation of IL-38 need to be further explored and could open new therapeutic opportunities for the treatment of arthritis.

\section{REFERENCE}

1. Boutet MA, et al. IL-38 overexpression induces anti-inflammatory effects in mice arthritis models and in human macrophages in vitro. Ann Rheum Dis 2017;76:1304-1312.

Disclosure of interest M. Harel: None declared, T. Garraud: None declared, B. Le Goff: None declared, F. Blanchard 
Grant/research support from: Inserm, the Arthritis Foundation, the French Society of Rheumatology, Pfizer and the Région Pays de la Loire (Bioregate)

\section{P061 TARGETING B-CELL ACTIVATING FACTOR (BAFF) IMPAIRS ECTOPIC LYMPHONEOGENESIS IN MURINE MODELS OF SJÖGREN'S SYNDROME}

IJ Campos*, ${ }^{2} \mathrm{~T}$ Slocombe, ${ }^{1} \mathrm{~S}$ Nayar, ${ }^{1} \mathrm{~V}$ lannizzotto, ${ }^{1} \mathrm{DH}$ Gardner, ${ }^{1} \mathrm{CD}$ Buckley, ${ }^{2} \mathrm{~A}$ Haynes, ${ }^{2} \mathrm{RB}$ Henderson, ${ }^{1} \mathrm{~F}$ Barone. ${ }^{1}$ Institute of Inflammation and Ageing, University of Birmingham, Birmingham; ${ }^{2}$ Immunoinflammation TAU, GSK, Stevenage, UK

\subsection{6/annrheumdis-2018-EWRR2018.80}

Introduction Tertiary lymphoid structures (TLS) characterised by germinal centre formation and B cell proliferation represent the histological hallmark of primary Sjögren's syndrome (pSS). However, the events preceding the formation of such ectopic structures and factors driving their persistence are unknown. Overexpression of BAFF, also known as B cell lymphocyte stimulator (BLyS), in pSS patients has been linked with the presence of autoreactive B cells and autoantibody production. ${ }^{1}$ Furthermore, in pSS salivary glands BAFF is associated with the expansion of specific B cell subsets, and with B cell repopulation post rituximab treatment. ${ }^{2}$

Objectives In this work we aimed to dissect the dynamics of B cell subsets within tertiary lymphoid structures following BAFF-targeted treatment in both inducible and chronic animal models that mimic the histological features of pSS.

Methods Submandibular salivary glands of C57BL/6 mice were intra-ductally cannulated with luciferase-encoding replicationdeficient adenovirus to induce TLS formation as previously described. $^{3}$ Prior to salivary gland cannulation, mice were treated with two doses (i.p.) of either anti-BLyS mAb or isotype control. Salivary glands were dissected at day 15 postcannulation and TLS formation in both groups was assessed. NOD.B10.H2b mice were similarly treated with anti-BLyS $\mathrm{mAb}$ at 26 weeks old and salivary gland infiltrates assessed 21 days later.

Results Histological analysis of salivary glands from anti-BLyS treated C57BL/6 animals unveiled severely compromised TLS formation. Post anti-BLyS treatment, salivary glands were infiltrated by $\mathrm{T}$ cell clusters but only few, and scattered, B cells were present, contrasting with fully developed and organised TLS in the salivary glands of mice treated with isotype control. Significantly lower numbers of B cells, particularly from the B2 subset, as well as plasmablasts, infiltrated salivary glands of anti-BLyS treated mice. However, treatment with anti-BLyS did not affect numbers of infiltrating $\mathrm{T}$ cells (both CD4 and CD8), proliferative $\mathrm{T}$ cells, or plasma cells in inflamed salivary glands. In a chronic setting, salivary glands from NOD.B10.H2b mice were also infiltrated by significantly lower numbers of B2 B cells following anti-BLyS treatment.

Conclusions Our data highlights BAFF as a key player in ectopic lymphoneogenesis during inflammation as well as a subset-specific role for BAFF in B cell maturation. Furthermore, these results support future studies of BAFF-targeted therapeutics in pSS.

\section{REFERENCES}

1. Pers, et al. Ann N Y Acad Sci 2005.

2. Pers, et al. Arthritis Rheum 2007.

3. Bombardieri, Barone, et al. Jl 2012.
Acknowledgements This research is funded by GSK.

Disclosure of interest J. Campos: None declared, T. Slocombe Employee of: GSK, S. Nayar: None declared, V. Iannizzotto: None declared, D. Gardner: None declared, C. Buckley: None declared, A. Haynes Employee of: GSK, R. Henderson Employee of: GSK, F. Barone: None declared

\section{P062 SIGNIFICANT DECREASE OF T-CELLS BUT NOT MACROPHAGES IN THE SYNOVIUM OF PATIENTS WITH ACTIVE RHEUMATOID ARTHRITIS AFTER TREATMENT WITH TOCILIZUMAB}

K Chatzidionysiou*, M Engström, E af Klint, A Hensvold, Al Catrina. Rheumatology Department, Karolinska Institute, Stockholm, Sweden

\subsection{6/annrheumdis-2018-EWRR2018.81}

Introduction Tocilizumab (TCZ) is an anti-IL6R monoclonal antibody approved for the treatment of Rheumatoid Arthritis (RA). There is limited data on synovial tissue histology changes.

Objectives The aim of this study was to evaluate the effect of TCZ on synovial cell populations and on citrullination.

Methods 15 patients with definite RA, according to ACR 1987 criteria, independent of disease duration, were included. Synovial biopsies were obtained before and after 8 weeks of treatment with TCZ from all patients. We evaluated by immunohistochemistry (IHC) expression of citrullinated proteins (CP) and protein arginine deiminase (PAD) enzymes in synovial tissue before and after treatment with TCZ (1325:C03, 1325:B09, PAD2, PAD4). Negative controls were used for each antibody. Expression of CD68, CD3, CD20 and CD55 was also evaluated. Evaluation of all IHC variables was performed by two blinded independent observers using a semiquantitative score on a $0-3$ scale (0, no staining; 1 , low amounts of staining; 2, moderate amounts of staining; 3 , high amounts of staining). Paired-wised Wilcoxon Signed Ranks Test was used to compare the median value before and after treatment.

\begin{tabular}{llll}
\multicolumn{4}{c}{ Abstract P062 Table 1 } \\
\hline & Basline & 12 weeks & Wilcoxonsigned ranks test \\
\hline DAS28 BL & $5.9(4.7-6.8)$ & $2.98(2.0-3.8)$ & 0.028 \\
SJC BL & $9(3-14)$ & $1(0-4.25)$ & 0.009 \\
TJC BL & $10(4-15)$ & $1(0-2.25)$ & 0.017 \\
ESR BL & $34(15-69)$ & $6(5-16)$ & 0.001 \\
CRP BL & $11(5-27)$ & $1(1-2)$ & 0.005 \\
CD68 & $2(1-3)$ & $2(0.75-3)$ & 0.41 \\
CD3 & $2(1-3)$ & $1(0-2)$ & 0.046 \\
CD55 & $2(2-3)$ & $1.5(1-2)$ & 0.05 \\
C03 (cit) & $1(0-1)$ & $0(0-1)$ & 0.025 \\
B09 (cit) & $2(1-2)$ & $2(1-2)$ & 0.20 \\
PAD2 & $3(2-3)$ & $2(1.5-3)$ & 0.16 \\
PAD4 & $2(2-3)$ & $2(2-3)$ & 0.37 \\
\hline
\end{tabular}

Results The median (IQR) age, disease duration, N. prior biologic DMARDs and DAS28 at baseline was 66 (58-79), 4 (113), 1 (0-2), 6 (5-7), respectively. 93\% were female, 53\% were RF + and 60\% ACPA+, 53\% had concomitant glucocorticoids and only $27 \%$ had concomitant conventional synthetic DMARDs. Significant reductions in DAS28, swollen and 